\title{
Absorption Spectroscopic Determination of Solubility of Alloxazine in Aqueous Solutions
}

\author{
Alfons Penzkofer \\ Faculty of Physics, University of Regensburg, Regensburg, Germany \\ Email: alfons.penzkofer@physik.uni-regensburg.de \\ Received 9 April 2015; accepted 8 June 2015; published 11 June 2015 \\ Copyright (c) 2015 by author and Scientific Research Publishing Inc. \\ This work is licensed under the Creative Commons Attribution International License (CC BY). \\ http://creativecommons.org/licenses/by/4.0/ \\ (c) (i) Open Access
}

\begin{abstract}
The solubility of alloxazine in aqueous solution at pH $4\left(10^{-4}\right.$ molar $\left.\mathrm{HCl}\right)$ and $\mathrm{pH} 10\left(10^{-4}\right.$ molar $\mathrm{NaOH}$ ) at room temperature is determined by absorption spectroscopic characterization. Knowledge of the solubility is needed for sample preparation and quantitative spectroscopic solution characterization. Samples of different in-weight concentration were prepared and absorption spectra were measured versus storage time. The solubility limit concentration $C_{\text {sol }}$ was determined by the crossing point of the linearized absorption coefficient dependences on in-weight preparation concentration below and above the solubility limit. Values of $C_{\mathrm{sol}}=9.05 \pm 1 \mu \mathrm{M}$ and $14.5 \pm$ $1 \mu \mathrm{M}$ were determined for alloxazine in aqueous solution at $\mathrm{pH} 4$ and $\mathrm{pH} 10$, respectively.
\end{abstract}

Keywords

Solubility Limit Concentration, Alloxazine, Isoalloxazine, Absorption Method, Aqueous Solutions

\section{Introduction}

The knowledge of the solubility limit of a solute (molecule) in a solvent (host) is of fundamental importance for the preparation, characterization and application of solutions. The solute may be in gaseous, liquid or solid form and the host may be in gaseous, liquid or solid form at the mixture preparation and operation temperature [1]. The solubility may be defined as the capacity of two or more substances to form spontaneously with one another a homogeneous molecular or colloidal dispersion without chemical reaction [1]. Methods of the experimental determination of the solubility of solids in liquids are reviewed in [1]-[3].

The molecule alloxazine (benzo[g]pteridin-2,4(1H,3H)-dion) (CAS number: 490-59-5, PubChem Substance ID 2277695) and its tautomeric form isoalloxazine (structural formulae are shown in Figure 1) are the core of the broad family of flavins of which prominent members are lumichrome, lumiflavin, riboflavin, flavin mononuc- 


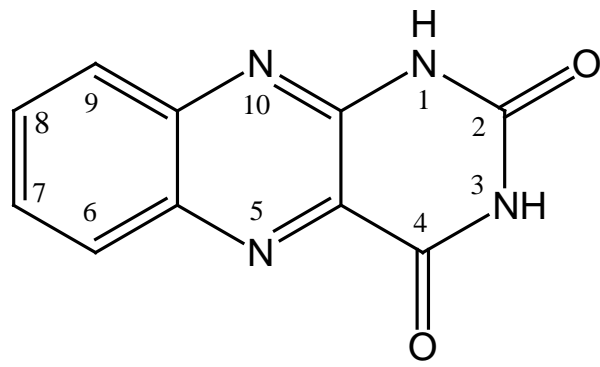

Alloxazine

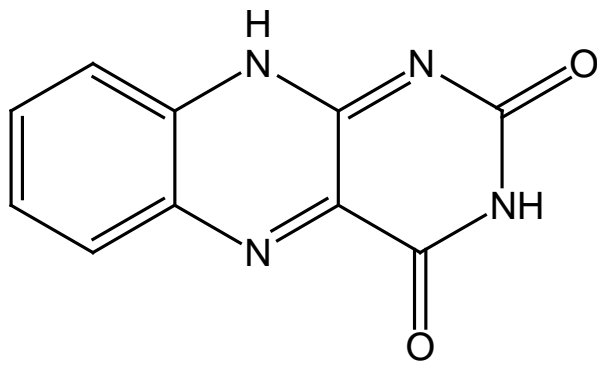

Isoalloxazine

Figure 1. Structural formulae of alloxazine and its tautomer isoalloxazine (see for example [11]). Sum formula: $\mathrm{C}_{10} \mathrm{H}_{6} \mathrm{~N}_{4} \mathrm{O}_{2}$. Molar mass: $214.18 \mathrm{~g} \cdot \mathrm{mol}^{-1}$.

leotide (FMN), and flavin adenine dinucleotide (FAD) [4]-[6]. In aqueous solution, the alloxazine form is expected to be presented at neutral and acidic $\mathrm{pH}$, and the isoalloxazine form is expected to be partly present at alkaline $\mathrm{pH}$ similar to the situation of lumichrome (7,8-dimethyl-alloxazine [7]-[9]. Alloxazine is poorly soluble in aqueous solution [10] and therefore has spectroscopically not been well characterized in water. The solubility (solubility limit concentration) of alloxazine in aqueous solutions has not yet been investigated in detail. Only in [10] a solubility concentration of $C_{\text {sol }}=15.5 \mu \mathrm{M}$ is reported for alloxazine in double-distilled water.

Here, the solubility of the solid organic dye alloxazine in the solvents $10^{-4}$ molar $\mathrm{HCl}$ in water and $10^{-4}$ molar $\mathrm{NaOH}$ in water at room temperature is determined by absorption spectra measurements. Samples of different inweight dye concentration were prepared, and the development of their absorption spectra with storage time was followed until steady-state equilibration was observed. Below the solubility limit, all the in-weighted dye eventually dissolves and the probe light absorption increases roughly linear with the amount of applied dye. Above the solubility limit, a part of the in-weighted dye does not dissolve and does not contribute to the probe light absorption.

\section{Experimental}

The molecular structures of alloxazine and its tautomer isoalloxazine [11] are displayed in Figure 1. The dye was purchased from Aldrich (purity 96\%) and used as delivered. The $\mathrm{pH} 4$ aqueous solvent was prepared by diluting $1 \mathrm{M} \mathrm{HCl}$ from Fluka to $1 \times 10^{-4} \mathrm{M} \mathrm{HCl}$ with Millipore water. The $\mathrm{pH} 10$ aqueous solvent was prepared by diluting $1 \mathrm{M} \mathrm{NaOH}$ from Merck to $1 \times 10^{-4} \mathrm{M} \mathrm{NaOH}$ with Millipore water. Solutions were prepared by inweighing an appropriate amount of alloxazine powder to the solvent and then subsequently taking aliquots and diluting them. Before taking aliquots the samples were shacked to get homogenous distributions. After each dilution step the samples were sonicated for $10 \mathrm{~min}$. Then fused silica cells (size $10 \times 10 \times 45 \mathrm{~mm}^{3}$ ) were filled with $4 \mathrm{~cm}^{3}$ of the prepared solutions and centrifuged for $15 \mathrm{~min}$ at $2000 \mathrm{rpm}$ in an Eppendorf Centrifuge $5702 \mathrm{R}$ at $4^{\circ} \mathrm{C}$. For the strongest diluted aliquots fused silica cell of $5 \mathrm{~cm}$ path length were filled up. These $5 \mathrm{~cm}$ cells were not centrifuged (could not be placed in the centrifuge). The prepared sample cells were kept at room temperature and their absorption spectra were daily measured over a time range of more than one week until no longer spectral changes were observed (complete dissolution for prepared in-weight concentration less than the solubility limit concentration, or equilibration between dissolved phase and un-dissolved solid phase for prepared in-weight concentration larger than the solubility limit concentration).

Transmission spectra measurements were carried out with a commercial spectrophotometer (Cary 50 from Varian). Attenuation coefficient spectra $\alpha(\lambda)$ were obtained from the transmission spectra $T(\lambda)$ by the relation $T(\lambda)=\exp [-\alpha(\lambda) \ell]$ where $\ell$ is the light path length through the sample. The attenuation coefficient spectra are composed of absorption and scattering contributions according to $\alpha(\lambda)=\alpha_{a}(\lambda)+\alpha_{s}(\lambda)$. The absorption coefficient spectra $\alpha_{a}(\lambda)$ were obtained by subtracting the scattering contribution $\alpha_{s}(\lambda)$. The scattering coefficient spectrum was determined by an empirical power law [12]-[14] of $\alpha_{s}(\lambda)=\alpha_{s}\left(\lambda_{0}\right)\left(\lambda_{0} / \lambda\right)^{\gamma}$ where $\lambda_{0}$ is a fixed wavelength in the transparency region of the solution (there $\left.\alpha\left(\lambda_{0}\right)=\alpha_{s}\left(\lambda_{0}\right)\right)$ and $\gamma$ is a fit parameter $(\gamma \leq 4$ depends on the scattering particle size with smaller $\gamma$ for larger particle size). In the figures below only absorption coefficient spectra $\alpha_{a}(\lambda)$ are shown. 


\section{Results}

\subsection{Alloxazine in Aqueous Solution at pH 4 (10-4 $\mathrm{M} \mathrm{HCl})$}

The temporal development of absorption coefficient spectra of alloxazine in $10^{-4} \mathrm{M} \mathrm{HCl}$ was studied for a series of in-weight concentrations $C$ spanning the range from below the solubility limit to above the solubility limit. The samples were kept at room temperature in the dark.

In Figure 2, the situation is depicted for the in-weight concentration of $C=6.89 \times 10^{-6} \mathrm{~mol} \cdot \mathrm{dm}^{-3}$ which is below the solubility limit (see below Figure 5). The absorption coefficient spectra are displayed for the storage times of $t=0$ (measurement immediately after preparation), $26.5 \mathrm{~h}, 50.5 \mathrm{~h}$, and $146.5 \mathrm{~h}$. The absorption increased with storage time. At $t=146.5 \mathrm{~h}$ the steady-state absorption situation was reached (no further rise of absorption with time, the dye was completely dissolved). The shape of the absorption coefficient spectra did not change with storage time.

In Figure 3, steady-state absorption coefficient spectra are shown (storage time $146.5 \mathrm{~h}$ ) for various in-weight concentrations in the range from $C=4.41 \times 10^{-7} \mathrm{~mol} \cdot \mathrm{dm}^{-3}$ (well below solubility limit) to $C=4.307 \times 10^{-4}$ $\mathrm{mol} \cdot \mathrm{dm}^{-3}$ (well above the solubility limit). At low in-weight concentrations the absorption increased roughly linear proportional with the concentration, and at high in-weight concentration the absorption became nearly concentration independent. The shapes of the absorption spectra are roughly concentration independent.

At the fixed wavelength of $\lambda=380 \mathrm{~nm}$ the storage time dependence of the absorption coefficient for the investigated in-weight concentrations is plotted in Figure 4. The data points are fitted by

$\alpha_{a}(t)=\alpha_{a}(0)+\Delta \alpha_{a}\left[1-\exp \left(-t / \tau_{\text {dis }}\right)\right]$, where $\alpha_{a}(0)$ is the absorption coefficient at the end of sample preparation (part of dye dissolved during preparation process), $\Delta \alpha_{a}=\alpha_{a}(\infty)-\alpha_{a}(0)$ is the absorption coefficient change during sample storage (part of dye powder slowly dissolves, or immediately excess dissolved dye re-aggregates and re-crystallizes), and $\tau_{d i s}$ is the time constant of dissociation equilibration. $\alpha_{a}(\infty)$ is the steady-state absorption coefficient. $\Delta \alpha_{a}$ changes from positive values at low concentration (continued dissolution) to negative values at high concentration (initial oversaturation). The inset in Figure 4 shows the relative absorption coefficient change $\Delta \alpha_{a} / \alpha_{a}(0)$ versus in-weight concentration $C$. The dissolution time constant varied with in-weight concentration in the range from $10 \mathrm{~h}$ to $40 \mathrm{~h}$.

In Figure 5, the steady-state absorption coefficient $\alpha_{a}(\infty)$ at $\lambda=380 \mathrm{~nm}$ is plotted versus the in-weight concentration $C$. At low concentration the absorption coefficient rises steeply with concentration, and then changes over to a weak concentration dependence with increasing concentration. The low-concentration absorption rise

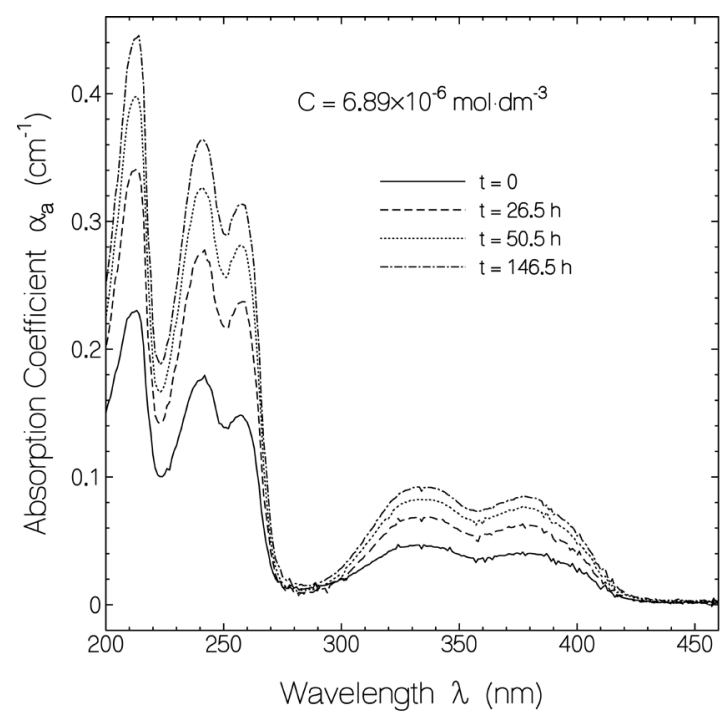

Figure 2. Temporal development of absorption coefficient spectra of $6.89 \mu \mathrm{M}$ alloxazine in aqueous solution at $\mathrm{pH} 4\left(10^{-4} \mathrm{M} \mathrm{HCl}\right)$. The sample was stored at room temperature $\left(21^{\circ} \mathrm{C} \pm 0.5^{\circ} \mathrm{C}\right) . t$ is the storage time after sample preparation. 


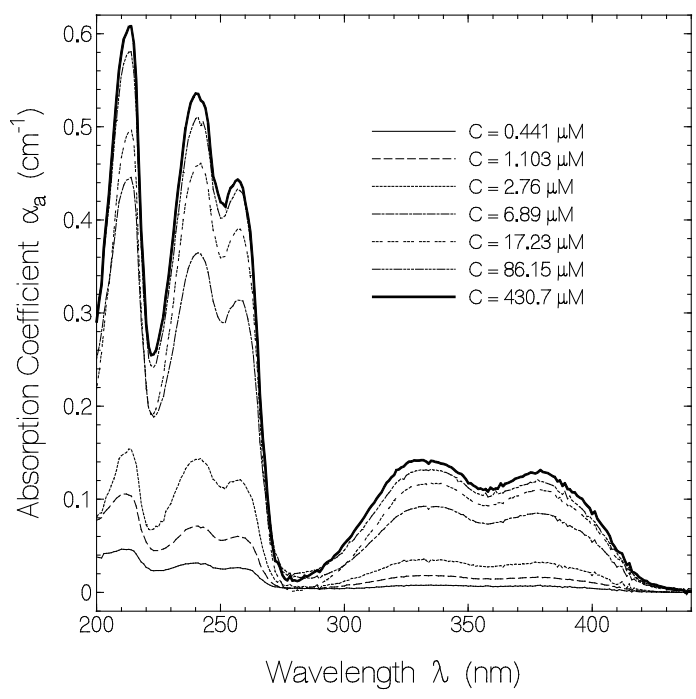

Figure 3. Concentration dependent absorption coefficient spectra of alloxazine in aqueous solution at $\mathrm{pH} 4$ at room temperature $\left(21^{\circ} \mathrm{C} \pm 0.5^{\circ} \mathrm{C}\right)$ after temporal equilibration. The storage time of the samples after preparation was $t=146.5 \mathrm{~h}$. The in-weight concentrations are listed in the legend.

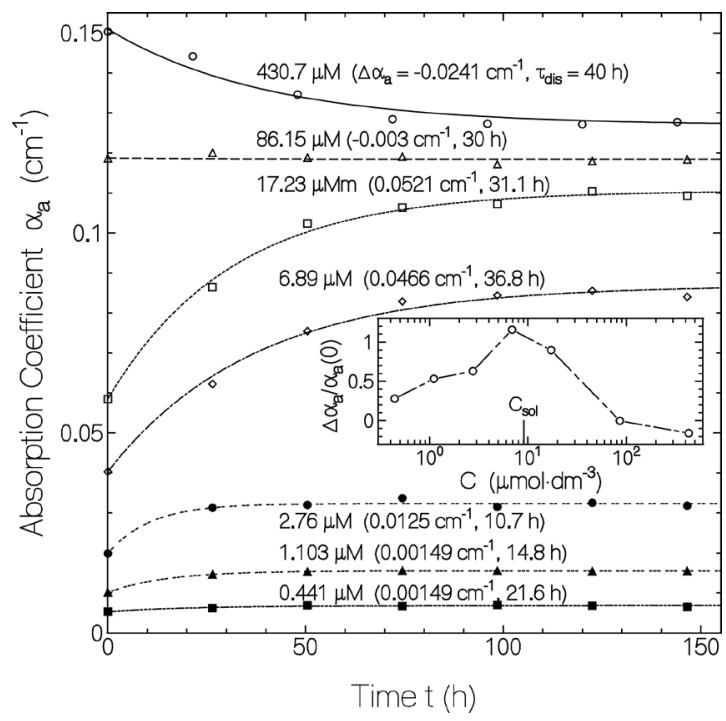

Figure 4. Temporal development of absorption coefficient $\alpha_{a}(380 \mathrm{~nm})$ of alloxazine in $\mathrm{pH} 4$ aqueous solution at room temperature for various in-weight concentrations. $t$ is storage time after sample preparation. The marks are measured and the curves are fits according to

$\alpha_{a}(t)=\alpha_{a}(0)+\Delta \alpha_{a}\left[1-\exp \left(-t / \tau_{\text {dis }}\right)\right]$. The fit parameters

$\Delta \alpha_{a}$ and $\tau_{\text {dis }}$ are listed at the curves in parenthesis. The inset displays $\Delta \alpha_{a} / \alpha_{a}(0)$ versus in-weight concentration.

and the high-concentration absorption dependence are linearized (dashed and dash-dotted lines). The in-weight concentration at the crossing point of the two linearized curves is defined as solubility limit concentration $C_{\text {sol }}$. The inset in Figure 5 shows an expansion of the low-concentration range with the linearized curves and the marked solubility limit concentration. A value of $C_{\text {sol }}=(9.05 \pm 1) \times 10^{-6} \mathrm{~mol} \cdot \mathrm{dm}^{-3}$ is extracted. 


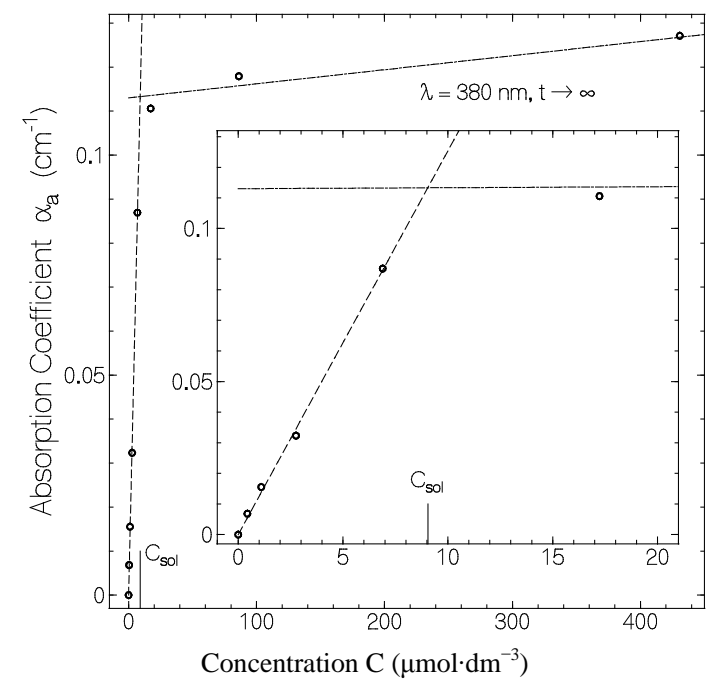

Figure 5. Extrapolated absorption coefficients $\alpha_{a}(t \rightarrow \infty$, $\lambda=380 \mathrm{~nm}$ ) of alloxazine in $\mathrm{pH} 4$ aqueous solution at room temperature displayed versus in-weight concentration. The solubility limit concentration $C_{\text {sol }}$ is determined by the crossing point of the linearized absorption coefficient dependences on concentration below and above the solubility limit. The inset presents an expansion of the figure at low concentration.

\subsection{Alloxazine in Aqueous Solution at pH 10 (10-4 $\mathrm{M} \mathrm{NaOH})$}

The temporal development of absorption coefficient spectra of alloxazine in $10^{-4} \mathrm{M} \mathrm{NaOH}$ was studied for a series of in-weight concentrations $C$ spanning the range from below the solubility limit to above the solubility limit. The samples were kept at room temperature in the dark.

In Figure 6 absorption coefficient spectra for several storage times are depicted for the in-weight concentrations of $C=7.824 \times 10^{-5} \mathrm{~mol} \cdot \mathrm{dm}^{-3}$ (top part, $C>C_{\text {sol }}$, see below) and $C=1.252 \times 10^{-5} \mathrm{~mol} \cdot \mathrm{dm}^{-3}$ (bottom part, $C$ $<C_{\text {sol }}$, see below). In the wavelength range $\lambda>420 \mathrm{~nm}$ an additional absorption band is seen which is attributed to isoalloxazine, the tautomeric form of alloxazine. Also the additional absorption band around $280 \mathrm{~nm}$ is attributed to isoalloxazine. Otherwise, below $420 \mathrm{~nm}$ the absorption is dominated by alloxazine. The isoalloxazine absorption changed stronger with storage time than the alloxazine absorption. For $C=7.824 \times 10^{-5} \mathrm{~mol} \cdot \mathrm{dm}^{-3}(C>$ $\left.C_{\text {sol }}\right)$ the alloxazine absorption decreased with storage time (initial excess dissolution). For $C=1.252 \times 10^{-5}$ $\mathrm{mol} \cdot \mathrm{dm}^{-3}\left(C<C_{\text {sol }}\right)$ the alloxazine absorption increased only slightly with storage time.

In Figure 7, steady-state absorption coefficient spectra are shown (storage time $169 \mathrm{~h}$ ) for various in-weight concentrations in the range from $C=8.01 \times 10^{-7} \mathrm{~mol} \cdot \mathrm{dm}^{-3}$ (well below solubility limit) to $C=4.89 \times 10^{-4}$ $\mathrm{mol} \cdot \mathrm{dm}^{-3}$ (well above the solubility limit). For $\lambda>420 \mathrm{~nm}$ the absorption is determined by isoalloxazine. Around $280 \mathrm{~nm}$ isoalloxazine contributions are seen. At low in-weight concentrations the alloxazine absorption increased roughly linear proportional with the concentration, and at high in-weight concentration the alloxazine absorption became nearly concentration independent. The steady-state isoalloxazine absorption changed less with in-weight concentration than the steady-state alloxazine absorption. The ratio of dissolved isoalloxazine concentration to alloxazine concentration increased with sample dilution.

In Figure 8, the absorption coefficient dependence at $\lambda=380 \mathrm{~nm}$ (dominant alloxazine absorption) on storage time is depicted for the investigated in-weight concentrations. Again the data points are fitted by $\alpha_{a}(t)=\alpha_{a}(0)+\Delta \alpha_{a}\left[1-\exp \left(-t / \tau_{\text {dis }}\right)\right]$. The inset in Figure 8 shows the relative absorption coefficient change $\Delta \alpha_{a} / \alpha_{a}(0)$ versus in-weight concentration $C$. The dissolution time constant $\tau_{\text {dis }}$ varied with in-weight concentration in the range from $16.4 \mathrm{~h}$ to $100 \mathrm{~h}$.

In Figure 9, the steady-state absorption coefficient $\alpha_{a}(\infty)$ at $\lambda=380 \mathrm{~nm}$ is plotted versus the in-weight concentration $C$. At low concentration the absorption coefficient rises steeply with concentration, and then changes 


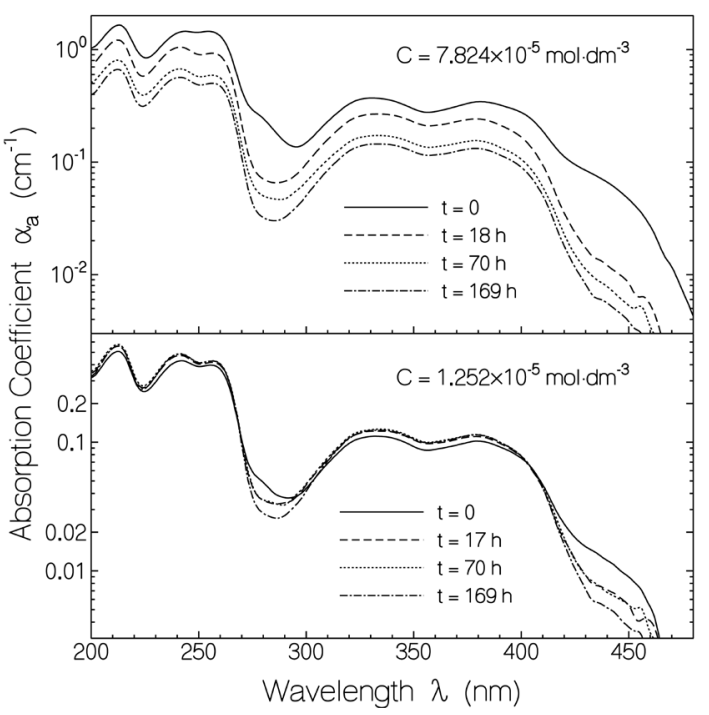

Figure 6. Temporal development of absorption coefficient spectra of $78.24 \mu \mathrm{M}$ (top part) and $12.52 \mu \mathrm{M}$ (bottom part) alloxazine in aqueous solution at $\mathrm{pH} 10\left(10^{-4}\right.$ $\mathrm{M} \mathrm{NaOH})$. The sample was stored at room temperature $\left(21^{\circ} \mathrm{C} \pm 0.5^{\circ} \mathrm{C}\right) . t$ is the storage time after sample preparation.

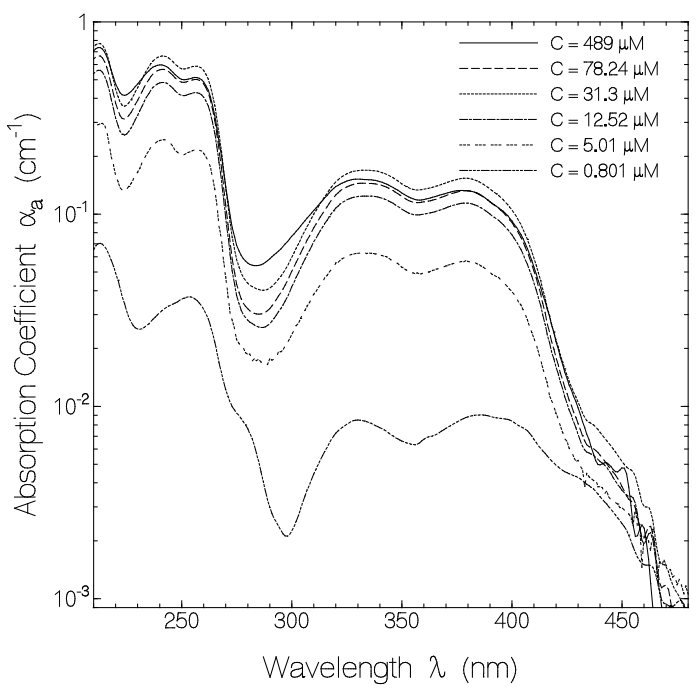

Figure 7. Concentration dependent absorption coefficient spectra of alloxazine in aqueous solution at $\mathrm{pH} 10$ at room temperature $\left(21^{\circ} \mathrm{C} \pm 0.5^{\circ} \mathrm{C}\right)$ after temporal equilibration. The storage time of the samples after preparation was $t=169 \mathrm{~h}$.

over to a weak concentration dependence with increasing concentration. The low-concentration absorption rise and the high-concentration absorption dependence are linearized (dashed and dash-dotted lines). The inset in Figure 9 shows an expansion of the low-concentration range with the linearized curves and the marked solubility limit concentration. A value of $C_{\text {sol }}=(1.45 \pm 0.1) \times 10^{-5} \mathrm{~mol} \cdot \mathrm{dm}^{-3}$ is extracted.

\section{Discussion}

The solubility of the organic molecule alloxazine in aqueous solution at $\mathrm{pH} 4$ (only fundamental form with pro- 


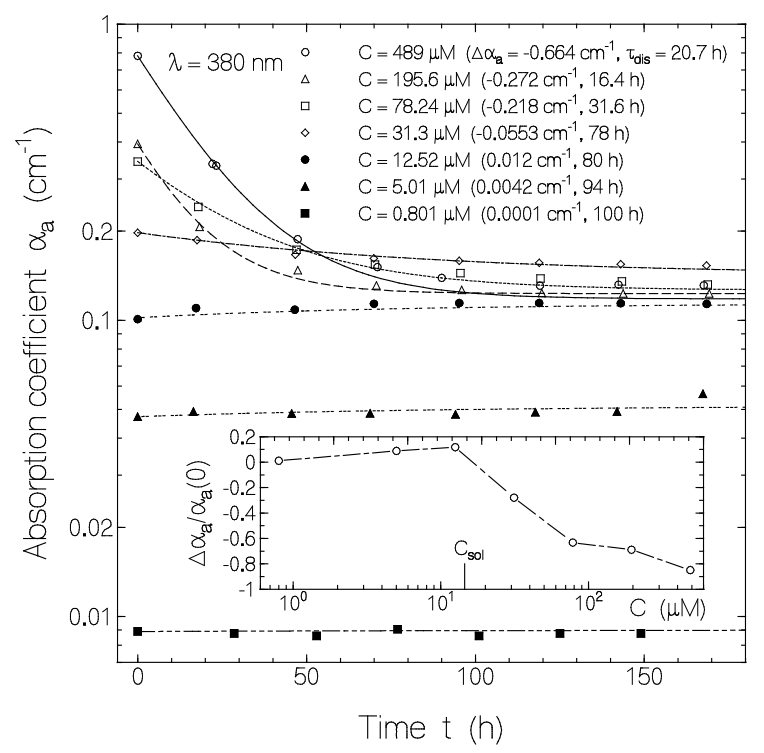

Figure 8. Temporal development of absorption coefficient $\alpha_{a}(380 \mathrm{~nm})$ of alloxazine in $\mathrm{pH} 10$ aqueous solution at room temperature for various in-weight concentrations. $t$ is storage time after sample preparation. The marks are measured and the curves are fits according to

$\alpha_{a}(t)=\alpha_{a}(0)+\Delta \alpha_{a}\left[1-\exp \left(-t / \tau_{\text {dis }}\right)\right]$. The fit parameters $\Delta \alpha_{a}$ and $\tau_{d i s}$ are listed at the curves in parenthesis. The fit includes $\alpha_{a}(380 \mathrm{~nm}, t=1176 \mathrm{~h})$ not shown in the figure. The inset displays $\Delta \alpha_{a} / \alpha_{a}(0)$ versus in-weight concentration $C$.

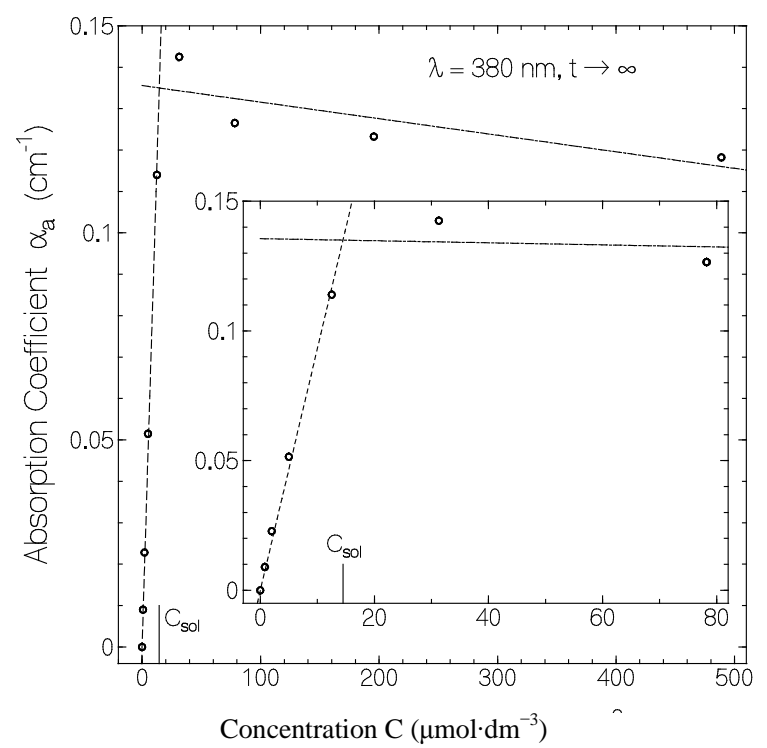

Figure 9. Extrapolated absorption coefficients $\alpha_{a}$ ( $t \rightarrow$ $\infty, \lambda=380 \mathrm{~nm}$ ) of alloxazine in $\mathrm{pH} 10$ aqueous solution versus in-weight concentration $C$. The solubility concentration is determined by the crossing point of the linearized absorption coefficient dependences on concentration below and above the solubility limit. The inset presents an expansion of the figure at low concentration. 
ton at $\mathrm{N} 1$ position) and $\mathrm{pH} 10$ (fundamental form and small fraction of tautomeric form isoalloxazine with proton at N10 position) was studied with an absorption spectroscopic method. Samples with rising in-weight concentration were prepared from well below to high above the solubility limit concentration.

For in-weight concentrations below the solubility limit the dissolution was not complete at the end of the preparation process (pouring solute in solvent, shaking, and sonication). The dissolution was only complete after tens of hours of keeping the solution undisturbed in the dark at room temperature.

For in-weight concentrations well above the solubility limit some oversaturation (excess dissolution) was observed at the end of the preparation process. The equilibrium solubility limit was approached on tens of hours time scale under undisturbed conditions in the dark at room temperature by re-aggregation, re-crystallization and sedimentation.

The in-weight concentration dependent initial dissolution depends on the sample preparation conditions (duration and intensity of shaking, stirring, sonication, preparation temperature) and on the solid solute composition (crystallinity, amorphous condition, porosity, particle size). But the steady-state solubility limit concentration at fixed temperature and fixed ambient conditions is thought to be constant independent of the preparation conditions.

The solubility limit concentration of alloxazine in aqueous solution at $\mathrm{pH} 4$ and $\mathrm{pH} 10$ were determined to be $C_{\text {sol }}(\mathrm{pH} 4)=9.05 \pm 1 \mu \mathrm{M}$ and $C_{\text {sol }}(\mathrm{pH} 10)=14.5 \pm 1 \mu \mathrm{M}$. These concentrations are small, but still large enough for applications of alloxazine in aqueous solutions and their absorption and emission spectroscopic characterization.

\section{Conclusions}

There exist many techniques in the literature [1]-[3] to determine the solubility of solid compounds in liquid hosts (residue weight, gravimetry, chemical analysis, conductometry, polarography, turbidimetry, refractometry and others).

The here described absorption spectroscopic method of steady-state absorption coefficient determination as a function of solid solute compound in-weight is widely applicable to solutes absorbing in a transparent spectral region of the liquid solvent. It may be applied over a wide temperature range between the melting and boiling point of the solvent. It is highly sensitive allowing the determination of the solubility limit of sparingly soluble solids in liquids. It may become the standard method for the solubility limit determination of organic and inorganic dyes (molecules absorbing somewhere between the near infrared and the near ultraviolet) in organic and inorganic liquid solvents.

\section{Acknowledgements}

The author is thankful to Prof. F. J. Gießibl for his kind hospitality. This work was supported by the German Research Foundation (DFG) within the funding program Open Access Publishing.

\section{References}

[1] Void, R.D. and Void, M.J. (1945) Determination of Solubility. In: Weissberger, A., Ed., Physical Methods of Organic Chemistry, Vol. 1, Interscience Publishers, Inc., New York, 107-133.

[2] Zimmerman, R.K. (1952) The Experimental Determination of Solubilities. Chemical Reviews, 51, 25-65.

[3] Cohen-Adad, R. and Cohen-Adad, M.-T. (2003) Solubility of Solids in Liquids. In: Hefter, G.T. and Tomkins, R.P.T., Eds., The Experimental Determination of Solubilities, Wiley Series in Solution Chemistry, Vol. 6, John Wiley \& Sons, Hoboken, 159-314. http://dx.doi.org/10.1002/0470867833.ch8

[4] Müller, F. (1991) Chemistry and Biochemistry of Flavoenzymes. Vol. 1, CRC Press, Boca Raton.

[5] Silva, E. and Edwards, A.M. (2006) Flavins: Photochemistry and Photobiology. Royal Society of Chemistry, London. http://dx.doi.org/10.1039/9781847555397

[6] Weber, S. and Schleicher, S.E. (2014) Flavins and Flavoproteins. Methods and Protocols, Series: Methods in Molecular Biology. Vol. 1146, Springer Verlag, Heidelberg. http://dx.doi.org/10.1007/978-1-4939-0452-5

[7] Tyagi, A. and Penzkofer, A. (2011) Absorption and Emission Spectroscopic Characterization of Lumichrome in Aqueous Solutions. Photochemisty Photobiology, 87, 524-533.

[8] Marchena, M., Gil, M., Martín, C., Organero, J.A., Sanchez, F. and Douhal, A. (2011) Stability and Photodynamics of 
Lumichrome Structures in Water at Different pHs and in Chemical and Biological Caging Media. Journal of Physical Chemisty B, 115, 2424-2435.

[9] Prukla, D., Sikorska, E., Koput, J., Khmelinskii, I., Karolczak, J., Gierszewski, M. and Sikorski, M. (2012) Acid-Base Equilibriums of Lumichrome and Its 1-Methyl, 3-Methyl, and 1,3-Dimethyl Derivatives. Journal of Physical Chemistry A, 116, 7474-7490.

[10] Terekhova, I.V., Kozbial, M., Kumeev, R.S. and Alper, G.A. (2011) Inclusion Complex Formation between Modified Cyclodextrins and Riboflavin and Alloxazine in Aqueous Solution. Journal of Solution Chemistry, 40, 1435-1446. http://dx.doi.org/10.1007/s10953-011-9724-0

[11] Salzmann, S. and Marian, C.M. (2009) The Photopysics of Alloxazine: A Quantum Chemical Investigation in Vacuum and Solution. Photochemical Photobiological Sciences, 8, 1655-1666. http://dx.doi.org/10.1039/b9pp00022d

[12] Penzkofer, A., Shirdel, J., Zirak, P., Breitkreuz, H. and Wolf, E. (2007) Protein Aggregation Studied by Forward Light Scattering and Light Transmission Analysis. Chemical Physics, 342, 55-63. http://dx.doi.org/10.1016/j.chemphys.2007.09.014

[13] Jagger, J. (1967) Introduction to Research in Ultraviolet Photobiology. Prentice Hall, Englewood Cliffs, 53.

[14] Oster, G. (1955) Light Scattering. In: Oster, G. and Pollister, A.W., Eds., Physical Techniques in Biological Research, Vol. 1, Optical Techniques. Academic Press, New York, 51-72. http://dx.doi.org/10.1016/b978-1-4832-3047-4.50008-1 